\title{
Interpretation of localized surface nano-structures
}

\author{
S A Morsi ${ }^{1}$, W M Moslem ${ }^{1,2}$, S K El-Labany ${ }^{3}$ \\ ${ }^{1}$ Department of Physics, Faculty of Science, Port Said University, Port Said, Egypt \\ ${ }^{2}$ Centre for Theoretical Physics, The British University in Egypt (BUE), El-Shorouk city, Cairo, Egypt \\ ${ }^{3}$ Department of Physics, Faculty of Science, Damietta University, New Damietta 34517, Egypt
}

\section{Article Info}

Received April 16, 2019

\section{Keyword:}

Plasma expansion

Surface nano-structure

Self-similar appraoch

Non-extensive distribution

\section{Corresponding Author:}

S A Morsi,

Department of Physics, Faculty of Science, Port Said University, Port Said, Egypt.

Email: S.morsi@sci.psu.edu.eg.

\begin{abstract}
Recent experiment was done to create surface nano-structures by extrusion of slow highly charged ions with LiNbO3 single crystal. In this paper, we suggest a mechanism based on plasma expansion approach to explain the formation of the surface nano-structures. Furthermore, the effects of the physical parameters of the non-Maxwellian plasma on the created structures are examined.
\end{abstract}

\section{Introduction}

Plasma expansion has a massive development because of its vital role in understanding the nonlinear behavior in many applications extended from laboratory to space plasmas [1-3]. Plasma expansion research started in 1954 by Lifshits and Landau [4]. Firstly, the plasma expansion discovered at laboratory, where the studies concerned on the formation of high temperature and density plasma by concentrating laser spots. When a laser irradiates a small volume of the target a rapid heating of electrons, leading to the diffusion of heat into the interior of the body. A widespread of experimental and theoretical studies were performed to explain the mechanism of such expansion [5]. Furthermore, plasma expansion exists in astrophysical phenomena such as in planetary rings, solar wind, and in inert objects as Titan moon and Venus [6-9]. During the last decade, slow highly charged ions (HCIs) was used as new tool to create surface nano-structures in different materials including conductors (HOPG [10], Au [11]), semiconductors ( $\mathrm{ZnO}$ [12], Si [13], SiO2 [14]), and insulators ( $\mathrm{LiF}$ [15], $\mathrm{CaF} 2$ [16], KBr [17]). Furthermore, many works were made to create nonostructures in different materials (e.g. Refs [18-20]). On the other hand, surface nano-structures consider as a promising new challenge for up-to-date nano-technological applications such as integrated optics, because of its large electro optical and non-linear optical coefficients. The good advantage of this method is that the nano-structures in the surface are created without chemical etching as in optical lithographic methods. Also, the modiffication occurs only in the topmost layers, which prevents any composition changes in the material bulk. Depending on the energy and type of ion beam, the size and shape of the created nano-structures can be controlled. Understanding the mechanism for creating surface nano-structures is of vital importance for the future applications. During recent years, plasma expansion approach was used as a possible mechanism to generate surface nano-structures in different materials (see Refs. [12,21-23]). During last decade the formation of surface nano-structures by plasma expansion induced by highly charged ions was investigated by Moslem and 
El-Said [14]. They used plasma expansion approach with suitable hydrodynamic equations to explain the creation mechanism of the surface nano-hillocks induced by HCIs. Numerical analysis reveal that within the nanoscale created plasma region, the increase of the temperature causes an increase of the self-similar solution validity domain, and consequently the surface nano-hillocks become taller. Furthermore, the presence of the negative (positive) nano-dust particles would lead to increase (decrease) the nano-hillocks height. Later, ElSaid et al. [12] studied the creation of hillock-like nanostructures on the surface of zinc oxide single crystals by irradiation with slow highly charged ions. It is found that at constant kinetic energy, the nanostructures were only observed after irradiation with ions of potential energies above a threshold between $19.1 \mathrm{keV}$ and $23.3 \mathrm{keV}$. The size of the nanostructures increases as a function of potential energy. Again a plasma expansion approach was used to explain the nanostructures creation. The numerical solutions showed that the surface nanostructures became taller with the increase of ionic temperature. The influence of charged cluster formation and the relevance of their polarity are examined. Recently, Moslem et al. [24] investigated the creation of surface nano-structures using of $30 \mathrm{MeV} \mathrm{C}_{60}$ cluster ions to create nano-hillocks of size larger than the ones produced by $\mathrm{GeV}$ monoatomic ions. The physical mechanism underlying the realization of such nanostructures was elucidated using a plasma expansion approach. Numerical analysis showed that increasing the ionic temperature (number density) ratios would lead to decrease (increase) the nano-hillocks height. ElSaid et al. [25] irradiated lithium niobate $\left(\mathrm{LiNbO}_{3}\right)$ single crystals with 150-keV slow highly charged xenon ions, which produce hillock-like nano-structures. Till now, no theoretical explanation to this experiment was made, which is the motivation of the present study. Therefore, the aim of this work is to use plasma expansion approach as a possible theoretical model to creat surface nano-structures of $\mathrm{LiNbO}_{3}$ single crystal as presented in Ref [25]. For this purpose, we use a hydrodynamic fluid model of the moving ions (i.e. $\mathrm{Li}, \mathrm{Nb}$, and $\mathrm{O}$ ) and non-Maxwellian electrons to describe the possible mechanism of the surface nano-structures formation.

\section{Theoretical Model}

To describe the possible mechanism of surface nano-structure, we consider cold, unmagnetized, collisionless four components plasma consists of positive ions "Li" and "Nb" (referred by +1 and +2 , respectively), negative ions "O" (referred by -), and electrons (referred by e).

$$
\begin{aligned}
& \frac{\partial n_{j}}{\partial t}+\frac{\partial n_{j} u_{j}}{\partial x}=0 \\
& m_{j} n_{j}\left(\frac{\partial}{\partial t}+u_{j} \frac{\partial}{\partial x}\right) u_{j} \pm e Z_{j} n_{j} \frac{\partial \emptyset}{\partial x}=0
\end{aligned}
$$

and the electrons are assumed to obey non-extensive distribution

$$
n_{e}=n_{e 0}\left[1+(q-1) \frac{e \emptyset}{k_{B} T_{e}}\right]^{\frac{q+1}{2(q-1)}} .
$$

The system of equations is closed by the neutrality condition

$$
Z_{+1} n_{+1}+Z_{+2} n_{+2}-Z_{-} n_{-}-n_{e}=0 \text {. }
$$

In Eqs. (1)-(4), $n_{j}, u_{j}$ are the fluid densities and velocities of jth species $(j=+1,+2$, and -), and $\emptyset$ is an electrostatic potential. The plasma variables in Eqs. (1)-(4) are normalized as follow: $n_{j}$ by the unperturbed ion density $n_{j}^{(0)}, u_{j}$ by the ion-acoustic speed $C_{s}=\left(\frac{k_{B} T_{e}}{m_{+1}}\right)^{1 / 2}$, and $\emptyset$ by $k_{B} T_{e} / e$, where $k_{B}$ is the Boltzmann constant, and $\mathrm{e}$ is an electron charge magnitude. The neutrality condition reads $\beta_{+1}+\beta_{+2}-\beta_{-}=1$, where $\beta_{+1}=Z_{+1} n_{+1}^{(0)} / n_{e}^{(0)}, \beta_{+2}=Z_{+2} n_{+2}^{(0)} / n_{e}^{(0)}$, and $\beta_{-}=Z_{-} n_{-}^{(0)} / n_{e}^{(0)}$. We suppose that the plasma fills the halfspace $\mathrm{x}>0$, Cs matches with the ion motion time scale. The expansion front is formed by part of the ions that are subsequently accelerated, and it is associated with density depletion. The region of decreasing density moves into the ambient plasma with the ion-acoustic speed. Employing the dimensionless self-similar variable $\xi=x / C_{s} t$, into Eqs. (1)-(4) we obtain the following set of normalized ordinary differential equations [26, 27]:

$$
\begin{gathered}
\left(V_{j}-\xi\right) \frac{d N_{j}}{d \xi}+N_{j} \frac{d V_{j}}{d \xi}=0 \\
\left(V_{j}-\xi\right) \frac{d V_{j}}{d \xi}+Z_{j} \delta_{j} \frac{d \varphi}{d \xi}=0
\end{gathered}
$$




$$
\begin{aligned}
& \chi \frac{d \varphi}{d \xi}+\frac{1}{N_{e}} \frac{d N_{e}}{d \xi}=0 \\
& \beta_{+1} \frac{d N_{+1}}{d \xi}+\beta_{+2} \frac{d N_{+2}}{d \xi}-\beta_{-} \frac{d N_{-}}{d \xi}-\frac{d N_{e}}{d \xi}=0
\end{aligned}
$$

where $=\frac{-q-1}{2+2(q-1) \varphi}, N_{j}, V_{j}$, and $\varphi$ are the normalized densities, velocities, and electric potential, respectively, $\delta_{+1}=1, \delta_{+2}=m_{+1} / m_{+2}$, and $\delta_{-}=m_{+1} / m$.

\section{Numerical Solution and Discussion}

Before studying the surface nano-structures formation, two points are of interest:

- Firstly we ensure that the plasma conditions are justified during the expanding process. The plasma system length " $L$ " (i.e., hillocks height) should be larger than the Debye length $\lambda_{D}$. From the experimental data, the mass density of $\mathrm{LiNbO} 3$ is $4.65 \mathrm{~g} / \mathrm{cm} 3$, so the number of atoms $\simeq 4 \times$ $10^{21} \mathrm{~cm}^{-3 .}$ The nano-hillocks height is $\simeq 2.5 \times 10-7 \mathrm{~cm}$, and time of hillocks formation is about few picoseconds. Using these parameters, we have $\mathrm{L} / \lambda_{\mathrm{D}} \approx 12$. Therefore, the required plasma condition is well satisfied.

- The self-similar solution predicts the main criteria of the expansion front, i.e. it cannot expect the exact location of the ion front at early times. This means that the characteristic length and time cannot be defined. On the other hand, describing the phenomena with $\mathrm{x}$ and $\mathrm{t}$ is not possible and the physical parameters (i.e. number density, velocity, and potential) are described from a moving frame with velocity Cs. For fixed time of expansion (as in this experiment) the position can be considered as $\xi$. However, solving a system of 8 equations is not straightforward, such that here we have used a selfsimilar approach, which is the main advantage of this method.

The numerical solutions of the fluid Eqs. (5)-(8) show the dependence of the normalized density " $N_{+2}$ ", velocity " $V_{+2}$ ", and potential " $\varphi$ "' on the nonextensive parameter " $q$ " and density ratios " $\beta_{j}$ ". Solving Eqs. (5)(8) numerically, we obtain the following results. Figure (1) explains the effect of nonextensive parameter " $q$ " on the profile of " $N_{+2}$ ", " $V_{+2}$ ", and " $\varphi$ " with the expansion domain " $\xi$ ". There is no significant influence of " $q$ " on the expansion domain for small $\xi$, and the behavior is linear, For long $\xi$, an exponential decay is happen of " $N_{+2}$ " until the expansion ends at $\xi \simeq 0.47$. Increasing the value of " $q$ ", increases the expansion domain, but the expansion decreases for " $q$ " $>1.5$. Physically, the nonextensive distribution is suitable to describe the nonMaxwellian system, i.e. the system is not in thermal equilibrium due to the large charge separation between electrons and ions. On the other hand, at the early stage of the expansion, the ions have not enough pressure gradient to follow the electrons. Increasing the parameter " $q$ " means that the deviation from the thermal equilibrium is strong except at $q=1$ which leads up to to Maxwellian distribution (this case is out the scope of the present work). Strong deviation from the thermal equilibrium leads to reduce the expansion domain since the cold ions cannot follow the faster electrons to maintain the quasi-neutrality. Thus, the expansion domain, velocity and electric potential shrink with the increase of $q$.

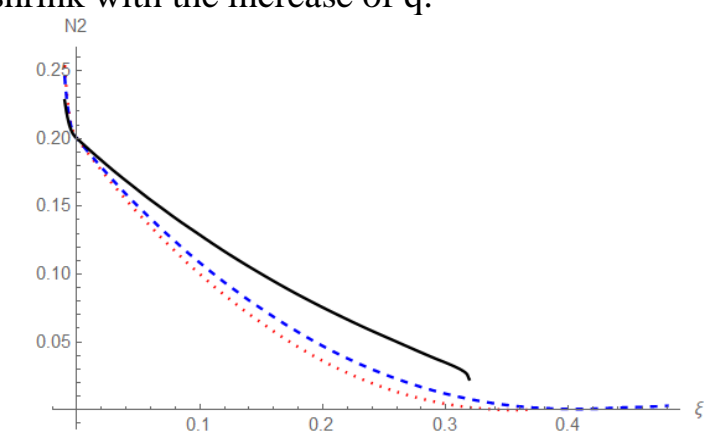

(a) 


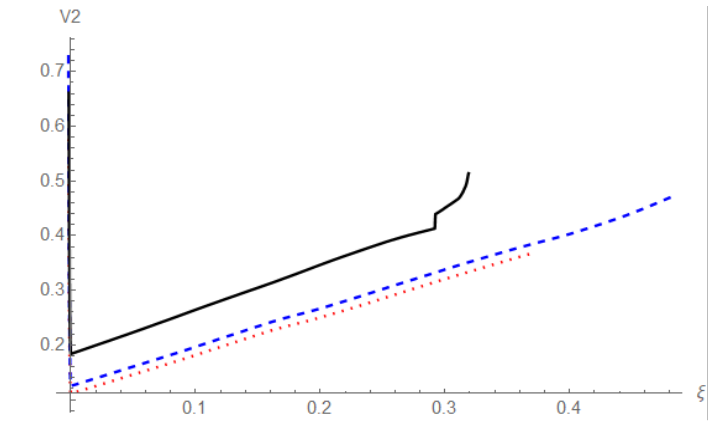

(b)

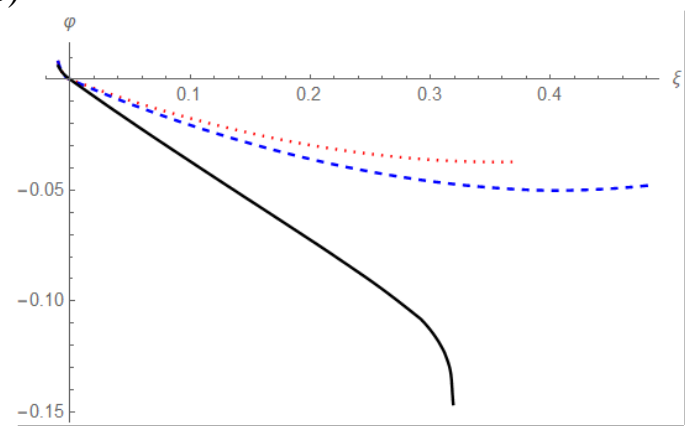

(C)

FIG. 1: (a) Normalized positive ion density " $\mathrm{N}_{+2}$ " (b) normalized positive ion velocity " $\mathrm{V}_{+2}$ ", and (c) normalized potential " $\varphi$ "' versus with dimensionless self-similar variable " $\xi$ " where $q=0.5$ (solid line), $q=1.5$ (dashed line), $q=2$ (dotted line). Here, $\beta_{+1}=0.5$ and $\beta_{+2}=0.2$.

The effects of density ratios $\beta_{+1}$ and $\beta_{+2}$ on the profile of " $N_{+2}$ ", " $V_{+2}$ ", and " $\varphi$ " are depicted in Figures (2) and (3). It is seen that there is no influence of the density ratio $\beta_{+1}$ at the early stage of the expansion domain until $\xi>0.05$. Increasing $\beta_{+1}$ and $\beta_{+2}$ lead to increase the expansion domain till the expansion stops creating the surface nano-hillocks structures. The ions velocity and electric potential increase with the increase of $\beta_{+1}$ and $\beta_{+2}$, they make the expansion domain increases. Therefore, the nano-hillocks height increase and become more towering.

(a)
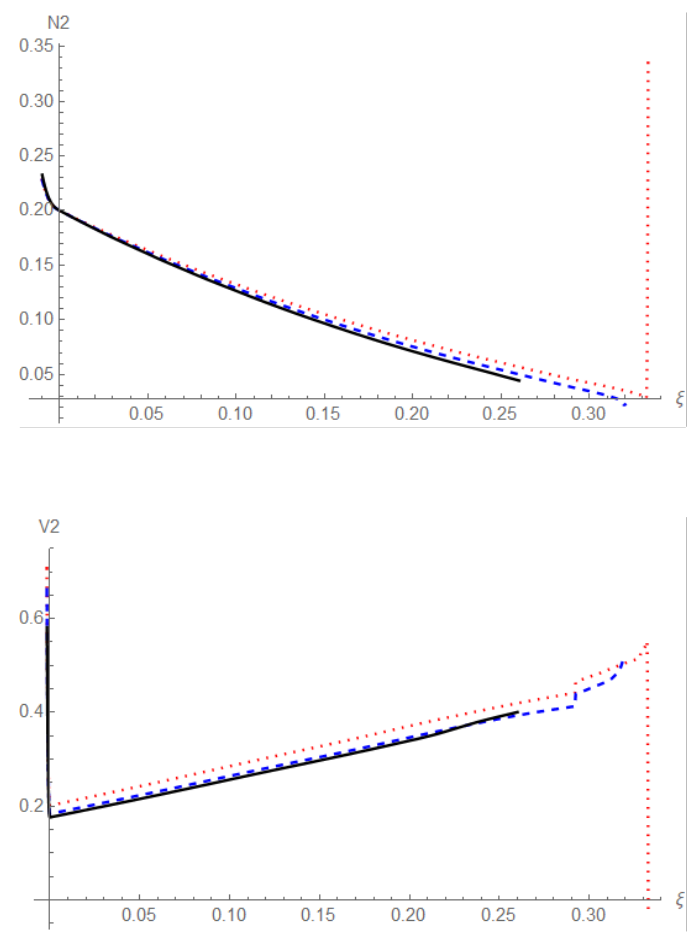

(b) 


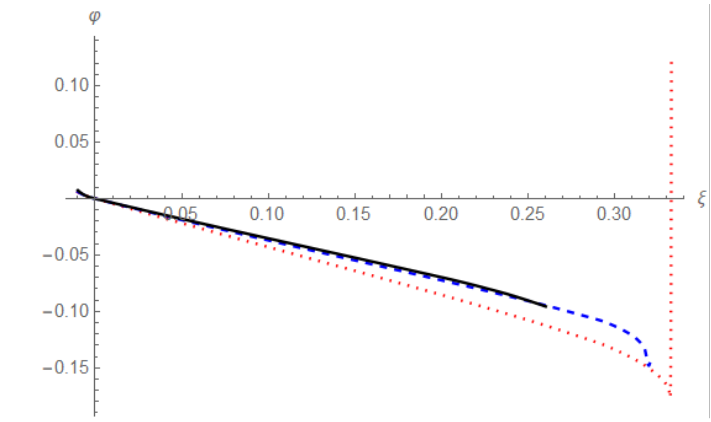

(c)

FIG. 2: (a) Normalized positive ion density " $\mathrm{N}_{+2}$ " (b) normalized positive ion velocity " $\mathrm{V}_{+2}$ ", and (c) normalized potential "' $\varphi$ " versus with dimensionless self-similar variable " $\xi$ " where $\beta_{+1}=0.4$ (solid line), $\beta_{+1}=0.5$ (dashed line), $\beta_{+1}=0.6$ (dotted line). Here, $q=0.5$ and $\beta_{+2}=0.2$.

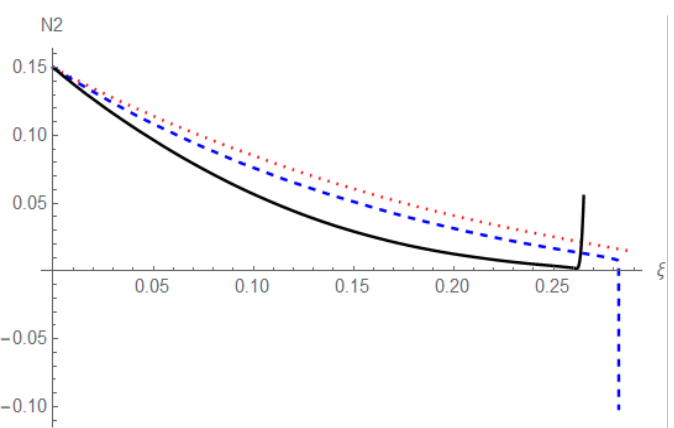

(a)

(b)
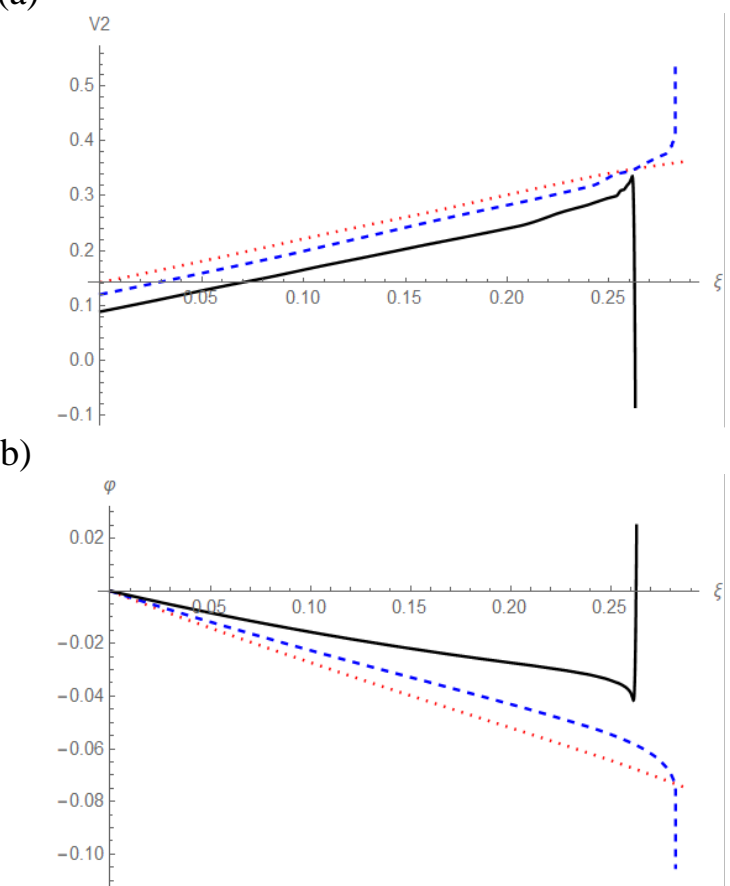

(c)

FIG. 3: (a) Normalized positive ion density " $\mathrm{N}_{+2}$ " (b) normalized positive ion velocity " $\mathrm{V}_{+2}$ ", and (c) normalized potential " $\varphi$ "' versus with dimensionless self-similar variable " $\xi$ " where $\beta_{+2}=0.05$ (solid line), $\beta_{+2}=0.1$ (dashed line), $\beta_{+2}=0.15$ (dotted line). Here, $q=0.5$ and $\beta_{+1}=0.5$.

\section{Summary}

To summarize, a plasma expansion approach is used to explain the creation mechanism of surface nano structure of $\mathrm{LiNbO}_{3}$ single crystals induced by HCIs. The fluid equations of cold positive and negative ions are used together with electrons obeying a non-extensive distribution. Numerical solutions of the basic 
equations provide us with the following results. Increasing the nonextensive parameter " $q$ " leads to increase the expansion domain until $q \geq 1.5$ then the expansion domain decreases. The nonextensive distribution is suitable to describe the system which is not in thermal equilibrium at the early stage of the expansion. Thus, the ions have not enough pressure gradient to follow the electrons and a large charge separation between electrons and ions is presented. Increasing $\beta_{+1}$ and $\beta_{+2}$ lead to grow the expansion domain and make the ions velocity faster, which increase the electric potential between the ions and electrons creating towering nanohillocks.

\section{References}

[1] Ch. Sack and H. Schamel, "Plasma expansion into vacuum A hydrodynamic approach", Phys. Rep., vol, 156, no. 6, pp. 311-395, 1987.

[2] U. R. I. Samir, K. H. Wright, Jr., and N. H. Stone, "The expansion of a plasma into a vacuum: Basic phenomena and processes and applications to space plasma physics", Rev. Geophys. Space Phys., vol. 21, no. 7, pp. 1631-1646, 1983.

[3] B. Doggett and J. G. Lunney, "Expansion dynamics of laser produced plasma", J. Appl. Phys., vol. 109, no. 9, pp. 3304, 2011.

[4] L. D. Landau, E. M. Lifshitz, M. Sploshnykh Sred, "Mechanics of Continuous Media", J. Appl. Math. Mech., vol. 26, no, 5, pp. 1293-1309, 1954.

[5] D. Bennaceur-Doumaz and D. Bara, "Self-similar solution of laser-produced plasma expansion into vacuum with kappa-distributed electrons", Nukleonika, vol. 61, pp. 115-118, 2016.

[6] M. S. Tiscareno and C. D. Murry, "Plentary Rings System", United Kingdom TJ International Ltd, Padstow, Cornwall, pp 517-530, 2018.

[7] A. V. Artemyev, V. Angelopoulos, and J. M. McTiernan, "Near-Earth solar wind: plasma characteristics from ARTEMIS measurements", Amer. Geophy.Union, 2018.

[8] S. Salem, W. M. Moslem, and A. Radi, "Expansion of Titan atmosphere", Phys. Plasmas, vol. 24, no. 5, pp 2901, 2017.

[9] H. Perez-de-Tejada, R. Lundin, Y. Futaana, T. L. Zhang, "Properties of planetward ion ows in Venus magnetotail", Icarus, vol. 274, no. 24, pp. 73-82, 2016.

[10] M. Sakurai, M. Tona, H. Watanabe, N. Nakamura, S. Ohtani, T. Terui, S. Mashiko, and H. A. Sakaue, "Development and applications of electron beam ion source for nanoprocesses", J. Phys. Conf. Ser., vol. 163, pp. 012115, 2009.

[11] C. H. Seager and A. F. Wright, "Role of carbon in GaN", J. Appl. Phys., vol. 92, no. 11, pp. 6553, 2002.

[12] A. S. El-Said, W. M. Moslem, and M. Djebli, "Surface nanostructuring by ion-induced localized plasma expansion in zinc oxide", Appl. Phys. Lett., vol. 104, pp. 231609, 2014.

[13] M. Tona and S. Ohtani, "Surface processes of highly charged ions", J. Phys: Conf. Ser., vol. 185, pp. 012046, 2009.

[14] A. S. El-Said, "Nanostructures created in $\mathrm{SiO} 2$ surface: A comparison between the impingement by slow highly charged ions and by swift heavy ions", Nucl. Instrum. Meth. B, vol. 282, pp. 63, 2012.

[15] A. S. El-Said, R. A. Wilhelm, R. Heller, M. Sorokin, S. Facsko, and F. Aumayr, "Surface Modifications with Highly Charged Ions", Phys. Rev. Lett., vol. 117, no. 126, pp. 101, 2016.

[16] A. S. El-Said, R. A. Wilhelm, R. Heller, S. Facsko, C. Lemell, G. Wachter, J. Burgduorfer, R. Ritter, and F. Aumayr, "Formation of surface nano-structures by plasma expansion induced by highly charged ions", Phys. Rev. Lett., vol. 109, no. 117, pp. 602, 2012.

[17] R. Heller, S. Facsko, R. A. Wilhelm, and W. Muller,"Defect Mediated Desorption of the $\operatorname{KBr}(001)$ Surface Induced by Single Highly Charged Ion Impact", Phys. Rev. Lett., vol. 101, pp. 096102, 2008.

[18] O. Y. Bozkurt, O. Ozbek, and A. R. Abdo, "The Effects of Nanosilica on Charpy Impact Behavior of Glass/Epoxy Fiber Reinforced Composite Laminates" Period. Engin. Nat. Sci. vol. 5, no. 3, pp. 322, 2017.

[19] O. Inayatullah and V. Sinnasamy, Condition Monitoring of the Uncoated Carbide Cutting Tool in Turning Process of the Aluminum Alloy 6061 via Vibration, Period. Engin. Nat. Sci. vol. 5, no. 3, pp. 298, 2017.

[20] J. Sutkovic and J. Jasarevic, "A review on Nanoparticle and Protein interaction in biomedical applications" Period. Engin. Nat. Sci. vol. 4, no. 2, pp. 34, 2016.

[21] W. M. Moslem and A. S. El-Said, "Formation of surface nano-structures by plasma expansion induced by highly charged ions", Phys. Plasmas, vol. 19, pp. 123510, 2012. 
[22] W. M. Moslem, A. S. El-Said, R. Sabry, A. Shalouf, S. K. El-Labany, H. Bahlouli, "Nonlinear phenomenon in nanostructures creation by fast cluster ions", Phys. Lett. A, vol. 381, pp. 102, 2017.

[23] M. Djebli, A. Kiouche, A. S. El-Said, and H. Bahlouli, "On the formation of surface nano structures induced by slow highly charged ions", Phys. Plasmas, vol. 24, pp. 072115, 2017.

[24] W. M. Moslem, A. S. El-Said, R. Sabry, A. Shalouf, S. K. El-Labany, and H. Bahlouli, "Nonlinear phenomenon in nanostructures creation by fast cluster ions", Phys. Lett. A, vol. 381, pp. 102, 2017.

[25] A. S. El-Said, R. A. Wilhelm, S. Facsko, and C. Trautmann, "Surface nanostructuring of LiNbO3 by high-density electronic excitations", Nucl. Instr. Meth. Phys. Res. B, vol. 315, pp. 265, 2013.

[26] P. Mora, "Plasma Expansion into a Vacuum", Phys. Rev. Lett., vol. 90, pp. 185002, 2003.

[27] W. M. Moslem, "Self-similar expansion of white dwarfs", Astrophys. Space Sci., vol. 342, pp. 351-355, 2012. 